\title{
Atención estomatológica de niños con microcefalia y bajo peso al nacer (1000 g). Descripción de un caso*
}

Dental Care of Children with Microcephaly and Low-Birth Weight (1000 g). Case Report

Cuidados estomatológicos de crianças com microcefalia e baixo peso ao nascer (1000 g).

Relato de caso

Fecha de recepción: 21/06/2019 | Fecha de aceptación: 19/10/2019

Diana Carolina Álvarez Correa ${ }^{\mathrm{a}}$

Universidad Nacional de Colombia. Bogotá, Colombia. dicalvarezco@unal.edu.co. https://orcid.org/0000-0003-1048-3168

\section{CARlos Andrés Rodríguez Delgado}

Universidad Nacional de Colombia. Bogotá, Colombia. caarodriguezde@unal.edu.co. https://orcid.org/0000-0002-0954-9994

\section{LYNA Rocío BOBADILLA TURRIAgo \\ Universidad Nacional de Colombia. Bogotá, Colombia. $\underline{\text { rbobadillat@unal.edu.co. }}$ https://orcid.org/0000-0003-3025-5568}

ÁNGELA LILIANA GRANDAS RAMÍREZ 
Universidad Nacional de Colombia. Bogotá, Colombia. algrandasr@unal.edu.co. https://orcid.org/0000-0002-5661-2853

*Descripción de caso.

${ }^{a}$ Correspondencia: dicalvarezco@ unal.edu.co

doi: https://doi.org/10.11144/Javeriana.uo38-81.aenm

Cómo citar: Álvarez Correa DC, Rodríguez Delgado CA, Grandas Ramírez AL. Atención estomatológica de niños con microcefalia y bajo peso al nacer (1000 g). Descripción de un caso. Univ Odontol. 2019 jul-dic; 38(81). https://doi.org/10.11144/Javeriana.uo38-81.aenm

\section{RESUMEN}

Antecedentes: Una de las alteraciones del neurodesarrollo en los niños con $<1500 \mathrm{~g}$ de peso al nacer es la parálisis cerebral espástica (PCE) que se asocia con microcefalia, epilepsia, discapacidad intelectual y retraso global del desarrollo. Objetivo: Describir el manejo estomatológico de un niño con cuadriparesia espástica. Descripción del caso: Para publicar este caso, se obtuvo autorización en consentimiento informado. El paciente era visto por un equipo multidisciplinario desde temprana edad pero solo ingresó al servicio de Estomatología Pediátrica de la Fundación Hospital Pediátrico de la Misericordia de Bogotá cuando tenía de 3 años 7 meses. El niño tenía antecedentes de prematuridad: 28 semanas; peso: $925 \mathrm{~g}$; talla: $34 \mathrm{~cm}$ : perímetro 
cefálico: $25 \mathrm{~cm}$. Diagnósticos sistémicos: PCE, microcefalia, epilepsia, discapacidad intelectual grave y retraso global del desarrollo. Examen físico: $9 \mathrm{~kg}$ de peso, $52 \mathrm{~cm}$ de estatura y $34 \mathrm{~cm}$ de perímetro cefálico. Examen extraoral: dolicocéfalo, perfil convejo, micrognatismo mandibular, apertura bucal 1,5 cm. Examen intraoral: caries de infancia temprana. Se realizó atención odontológica integral con retiro de focos sépticos, motivación e instrucción en higiene oral. Conclusión: El equipo multidisciplinario para la atención de niños prematuros con alteraciones del neurodesarrollo, microcefalia y bajo peso al nacer debe contar con la presencia del(la) estomatólogo(a) u odontólogo(a) pediatra para iniciar procesos de promoción y prevención en salud bucal individualizados para evitar caries de infancia temprana y mejorar la calidad de vida.

\title{
Palabras clave
}

cuadriparesia; discapacidad y odontología; estomatología pediátrica; microcefalia; peso al nacer; odontología; odontología pediátrica; salud bucal; tratamiento multidisciplinario

\begin{abstract}
Background: One of the neurodevelopmental disorders in children with $<1500 \mathrm{~g}$ of birth weight is spastic cerebral palsy (SCP) that is associated with microcephaly, epilepsy, intellectual disability, and global developmental delay. Objective: To describe the dental management of a boy with spastic quadriparesis. Case description: To publish this case, authorization was obtained through informed consent signing. The patient was been seen by an multidisciplinary team since early age but was only sent to the Pediatric Dental Service of the Mercy Pediatric Hospital Foundation in Bogotá at 3 years 7 months of age. The boy had history of prematurity: 28 weeks;
\end{abstract}


weight: $925 \mathrm{~g}$; height: $34 \mathrm{~cm}$ : head circumference: $25 \mathrm{~cm}$. Systemic diagnoses: SCP, microcephaly, epilepsy, severe intellectual disability, and overall developmental delay. Physical exam: $9 \mathrm{~kg}$ weight, $52 \mathrm{~cm}$ height and $34 \mathrm{~cm}$ head circumference. Extraoral examination: dolichocephalous, convex profile, mandibular micrognathism, oral opening $1.5 \mathrm{~cm}$. Intraoral exam: early childhood caries. Comprehensive dental care was performed with removal of septic foci, motivation, and oral hygiene instruction. Conclusion: The multidisciplinary team for the care of premature children with neurodevelopmental disorders, microcephaly, and low birth weight must include a pediatric dentist to initiate personalized oral health promotion and prevention processes to avoid early childhood caries and improve quality of life.

\section{Keywords}

birth weight; comprehensive care; dentistry; disability and dentistry; microcephaly; oral health; pediatric dentistry; quadriplegia

\section{SUMÁRIO}

Antecedentes: Um dos distúrbios do desenvolvimento neurológico em crianças com $<1500 \mathrm{~g}$ de peso ao nascer é a paralisia cerebral espástica (PCE) associada a microcefalia, epilepsia, deficiência intelectual e atraso global do desenvolvimento. Objetivo: Descrever o tratamento estomatológico de um menino come quadriparesia espástica. Descrição do caso: Para publicar este caso, a autorização foi obtida mediante consentimento informado. O paciente foi atendido por uma equipe multidisciplinar desde tenra idade, mas só entrou no serviço de Estomatologia Pediátrica da Fundação Hospitalar Pediátrica da Misericórdia de Bogotá quando tinha 3 anos e 7 meses. O menino 
tinha uma história de prematuridade: 28 semanas; peso: $925 \mathrm{~g}$; tamanho: $34 \mathrm{~cm}$ : circunferência da cabeça: 25 cm. Diagnósticos sistêmicos: PCE, microcefalia, epilepsia, deficiência intelectual grave e atraso geral no desenvolvimento. Exame físico: $9 \mathrm{~kg}$ de peso, $52 \mathrm{~cm}$ de altura e $34 \mathrm{~cm}$ de perímetro cefálico. Exame extra-oral: dolicocefalia, perfil convexo, micrognatismo mandibular, abertura oral de $1,5 \mathrm{~cm}$. Exame intraoral: cárie na primeira infância. Foi realizado tratamento odontológico abrangente, com remoção de focos sépticos, motivação e instrução em higiene bucal. Conclusão: A equipe multidisciplinar de atendimento a crianças prematuras com distúrbios do desenvolvimento neurológico, microcefalia e baixo peso ao nascer deve ter a presença do dentista pediátrico para iniciar os processos de promoção e prevenção à saúde bucal individual para evitar cáries na primeira infância e melhorar a qualidade de vida.

\section{Palavras chave}

microcefalia; deficiência e odontologia; odontologia; odontologia pediátrica; peso ao nascer; quadriplegia; saúde bucal; tratamento abrangente

\section{INTRODUCCIÓN}

El "Informe de Acción Global sobre el Nacimiento Prematuro" de 2012 indica que en el mundo nacen 15 mil niños pretérmino. Entre ellos, aproximadamente $5 \%$ nace con $<28$ semanas de desarrollo (prematuros muy extremos), $15 \% 28-<32$ semanas (prematuros extremos) y $20 \% 32-$ $<37$ semanas (prematuros moderados). Además de ser pretérmino, los recién nacidos pueden

presentar bajo peso al nacer, esto es, <2.500 g (bajo peso al nacer) <1.500 g (muy bajo peso al nacer) y <1.000 g (najo peso extremo) (1). Una de las alteraciones del neurodesarrollo más 
frecuentemente encontradas en los niños pretérmino y con bajo peso extremo al nacer es la parálisis cerebral espástica, que se asocia a microcefalia, epilepsia, discapacidad intelectual severa y retraso global del desarrollo y del lenguaje entre $5 \%$ y $30 \%$ de los casos $(1,2)$.

La insuficiencia motriz de origen central (IMOC), anteriormente denominada parálisis cerebral (PC), se define como un "grupo de trastornos del desarrollo del movimiento y la postura, que causan limitación de la actividad, y se atribuyen a trastornos no progresivos que se producen en el cerebro del feto o del bebé en desarrollo". Los trastornos motores de la parálisis cerebral pueden estar acompañados de alteraciones en la sensibilidad, cognición, comunicación, percepción y comportamiento, y trastorno convulsivo (3).

La IMOC se puede clasificar según el tipo motor, la distribución topográfica y la capacidad motora funcional. Ahora bien, la cuadriparesia espástica es el tipo de IMOC más común que se caracteriza por rigidez muscular excesiva, considerada como un síntoma complejo que abarca diferentes discapacidades (3). La cuadriparesia espástica se presenta generalmente en recién nacidos con bajo peso para su edad gestacional. Es la más grave y representa entre $10 \%$ y $40 \%$ de la PC. En la mayoría de los casos, el índice de crisis epilépticas es alto (90\%) y generalmente los niños presentan un marcado retraso psicomotor, microcefalia (perímetro cefálico alterado), alteraciones visuales y auditivas (4). Ardila y Espinosa (5) indicaron que, en una población colombiana, la cuadriparesia espástica fue el hallazgos clínico más frecuente entre las PC en niños con microcefalia (5). 
En Latinoamérica, Nazer y Cifuentes (6), en el Estudio Colaborativo Latino Americano de Malformaciones Congénitas, definieron la microcefalia como "un cráneo congénito pequeño, definido por un perímetro cefálico por debajo de $3^{\circ}$ de la desviación estándar en las curvas apropiadas para edad y sexo". En un estudio de prevalencia de malformaciones congénitas en Latinoamérica realizado entre 1995 -2008, se observó que Brasil y Chile eran los países con mayor tasa de nacidos vivos con malformaciones corporales y que con el pasar del tiempo han ido aumentando en todos los países. La microcefalia en dicho informe presentó un promedio de 3,7 casos por cada 10.000 nacimientos para Latinoamérica y para Colombia la tasa fue de 3,4 por cada 10.000 nacimientos. Particularmente en la ciudad de Cali en Colombia, entre 2004 y 2008 se describieron 5 casos en el Hospital Universitario del Valle con una tasa de 1,52 casos por cada 10.000 nacidos (7).

Abanto et al. (8) comentan que los estudios muestran que, cuanto más grave es el daño neurológico, mayor es el riesgo de manifestaciones orales. Hay una alta incidencia de mordida abierta anterior $(9,10)$, alteraciones de la articulación temporomandibular, sialorrea, bruxismo, defectos del desarrollo del esmalte, trauma dentoalveolar y caries dental (10). El objetivo de este artículo es describir un caso de bajo peso al nacer y microcefalia, y destacar la importancia del(la) estomatólogo(a) u odontólogo(a) pediatra en el equipo multidisciplinario para promover la salud bucal y prevenir la caries de la infancia temprana.

\section{DESCRIPCIÓN DEL CASO}


Un niño de 3 años y 7 meses de edad ingresó al servicio de salud oral del Posgrado de Estomatología Pediátrica y Ortopedia Maxilar de la Universidad Nacional de Colombia, en la clínica de personas con discapacidad de la Fundación Hospital Pediátrico de la Misericordia. El motivo de consulta de los padres fue: "Tiene este diente dañado. Se lo han arreglado dos veces pero se le cae la calza. Nos dijeron que lo trajéramos acá”. Para publicar la descripción del caso y efectuar el tratamiento estomatológico se obtuvo el consentimiento informado del padre.

\section{Antecedentes}

Entre los antecedentes psicosociales se encontró que el niño vivía con sus padres, cuya actitud hacia el tratamiento era de colaboración. Con respecto a los antecedentes odontológicos, los padres indicaron que le habían realizado una resina dos veces y se le había caído. En cuanto a la dieta, el niño ingería 6 comidas o bebidas al día (diferentes a agua), tomaba biberón con leche en el día y la noche, se cepillaba los dientes 2 veces al día con crema dental sin flúor y no usaba seda dental.

Con respecto a los antecedentes prenatales, la madre tenía 27 años de edad al momento de la consulta y había sufrido diabetes gestacional y preclamsia grave. La descripción de los antecedentes perinatales incluyen cesárea de urgencia, nacimiento con un peso de $925 \mathrm{~g}$, una talla de $34 \mathrm{~cm}$ y un perímetro cefálico de $25 \mathrm{~cm}$. En cuanto a los antecedentes postnatales, el niño pasó 91 días en la unidad de cuidados intensivos, 60 días con ventilación mecánica, presentó hemorragia de la matriz germinal grado 2 derecha y grado 4 izquierda, microcefalia con acabalgamiento de suturas y epilepsia. Fue dado de alta en marzo de 2012 con un peso de $1930 \mathrm{~g}$, una talla de 45,5 cm y un perímetro cefálico de $27 \mathrm{~cm}$. En junio de 2012, el niño fue hospitalizado con diagnóstico de infección respiratoria por influenza tipo A, dificultad respiratoria moderada, neumopatía 
crónica y displasia broncopulmonar, microcefalia más cabalgamiento de suturas, epilepsia sintomática, desnutrición proteico-calórica, limitación de los esfuerzos terapéuticos, retraso global del neurodesarrollo, cuadriparesia espástica y alteración de la succión (deglución). Se le dio de alta en julio de 2012 y se mantuvo con oxígeno domiciliario, sin convulsiones y con fenobarbital $20 \mathrm{mg}$ cada 12 horas y topiramato $15 \mathrm{mg} /$ día.

\section{Examen clínico}

El niño presentó un perímetro cefálico de $34 \mathrm{~cm}$, con un peso de $9,2 \mathrm{~kg}$ y una talla de $82 \mathrm{~cm}$. De acuerdo con las tablas de la Organización Mundial de la Salud (OMS) de 2009, la puntuación Z mostraba bajos peso y talla por debajo del percentil 3 (figura 1). Durante el examen clínico general, el niño se encontraba alerta, consciente y orientado. Su pierna derecha era más corta que la izquierda y presentaba clinodactilia (curvatura) del quinto dedo de la mano. La cara tenía forma triangular, frente prominente, ojos grandes almendrados, nariz invertida, tercio inferior facial disminuido, asimetría facial del lado derecho, labios deshidratados, delgados y finos, comisuras labiales hacia abajo, micrognatia y mentón pequeño, perfil cóncavo, retrognatismo mandibular y clase II esquelética determinada clínicamente (figura 2).

FIGURA 1

TABLAS DE PATRONES DE CRECIMIENTO INFANTIL DE LA OMS DEL PRESENTE CASO
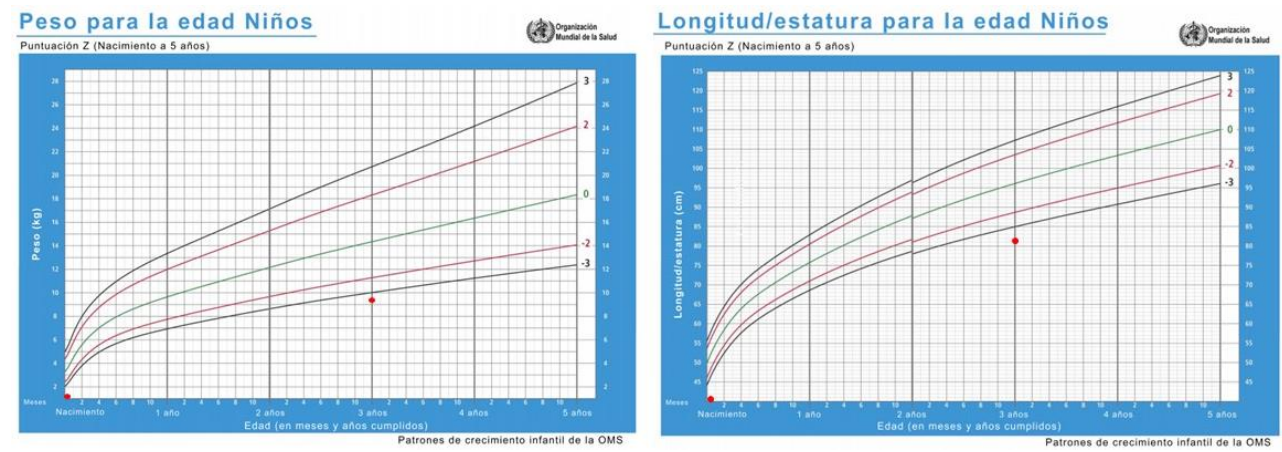

Fuente: Elaboración a partir de archivo de la historia clínica 
FIGURA 2

\section{FOTOS EXTRAORALES}

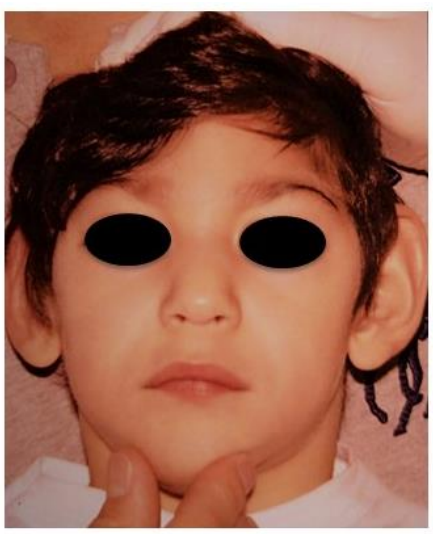

Frente

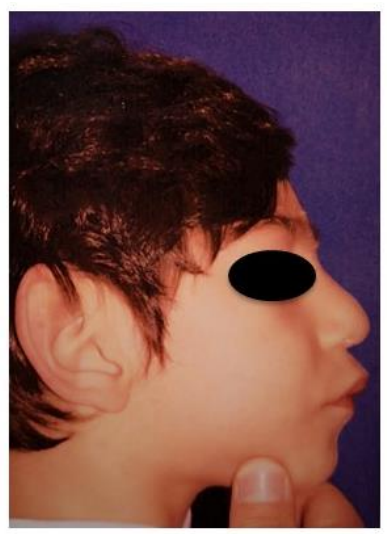

Perfil derecho

Fuente: Archivo de historia clínica

FIGURA 3

\section{FOTOS INTRAORALES}

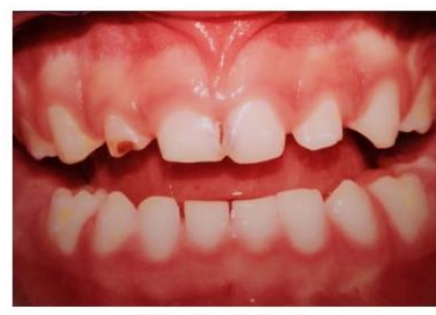

Oclusión de frente

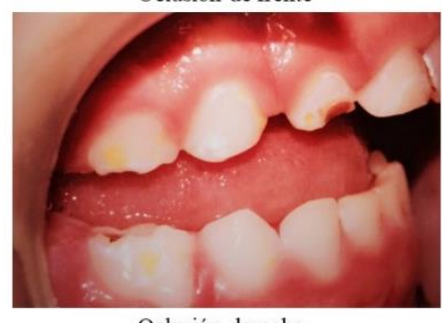

Oclusión derecha

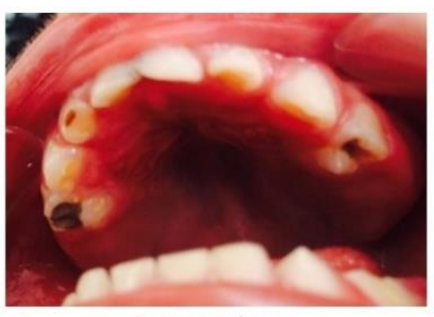

Arco superior

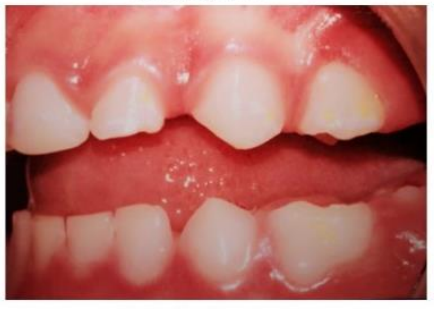

Oclusión Izquierda

Fuente: Archivo de historia clínica 


\section{Ayudas diagnósticas}

Se solicitaron apoyos diagnósticos tipo radiografía de perfil. El padre sostuvo la cabeza del niño para realizar el examen debido a, cómo podía esperarse por la cuadriparesia espástica, no había sostén cefálico. No fue posible analizar el tamaño ni la posición de los maxilares (figura 4).

FIGURA 4

RADIOGRAFÍA DE PERFIL

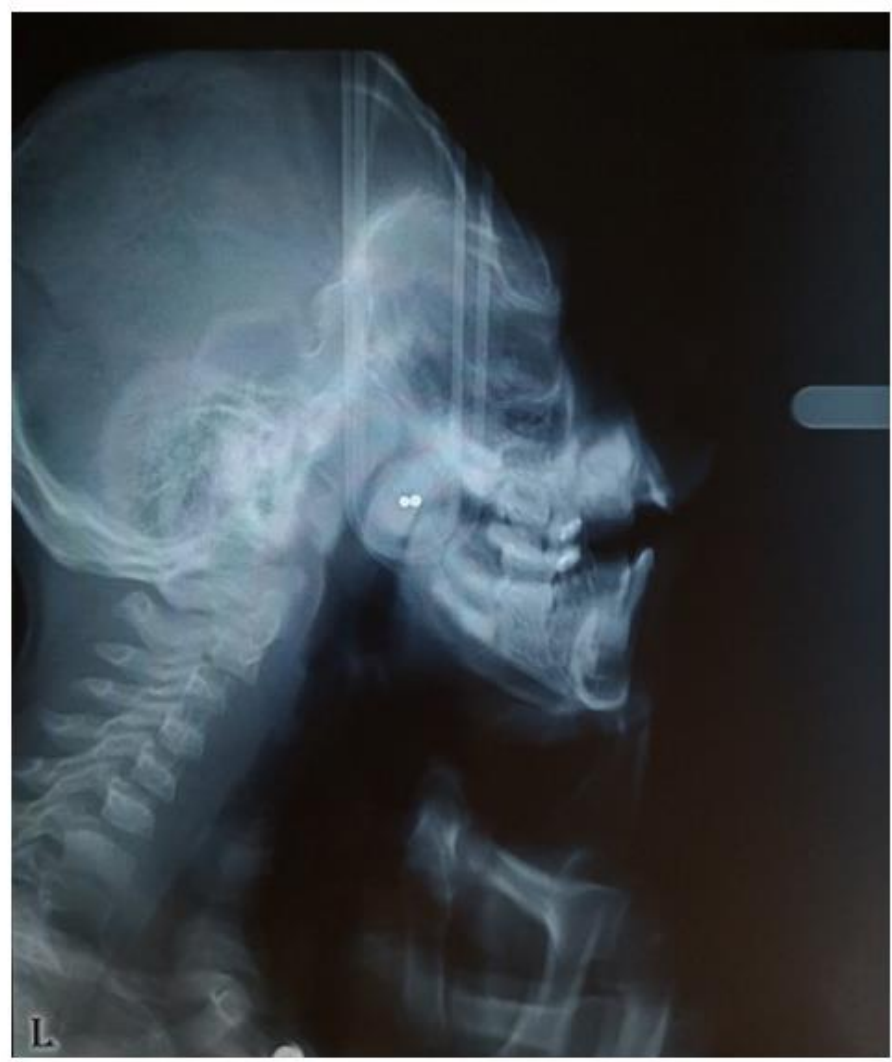

Radiografía de perfil

Fuente: Archivo de historia clínica

Asimismo, se indicó la toma de juego periapical para determinar el tratamiento dental y verificar posibles compromisos pulpares o alguna patología de furca (figura 5). 


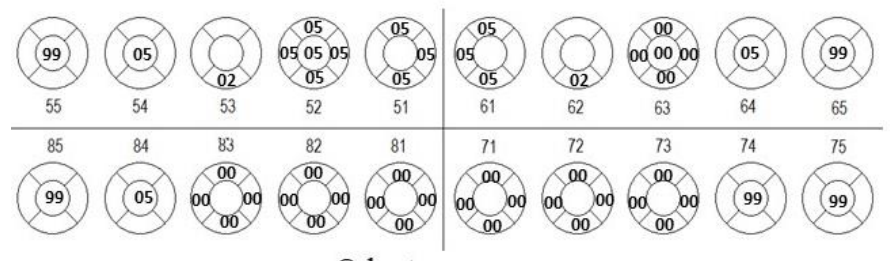

Odontograma
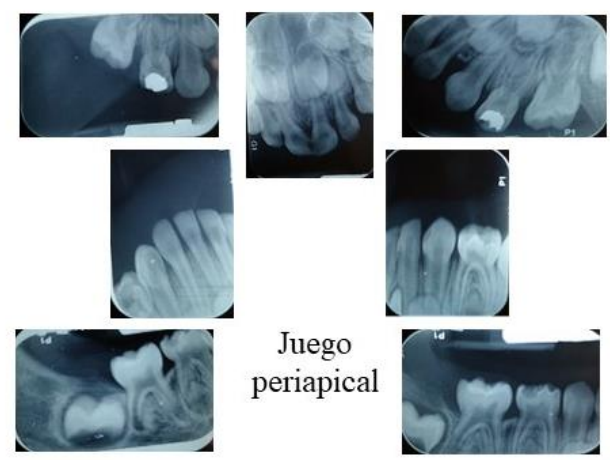

Fuente: Archivo de historia clínica

\section{Diagnóstico y objetivos del tratamiento}

En la figura 6 se describen los diagnósticos sistémico y orales. Entre de los objetivos del tratamiento estomatológico se establecieron:

1. Consejería dietaria y de hábitos

2. Adaptación a la consulta odontológica

3. Educación en higiene oral a los padres

4. Retiro de focos infecciosos

5. Restablecimiento de estética y función

FIGURA 6

DIAGNÓSTICOS SISTÉMICOS Y ORALES
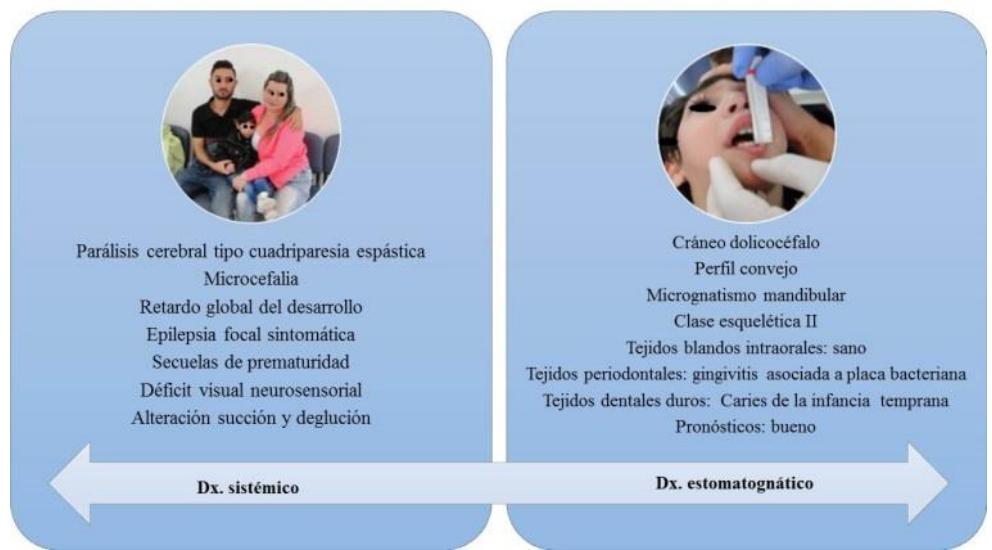


\section{Tratamiento}

El tratamiento del paciente se basó en un programa individual de valoración de riesgo de caries, consejería dietaria, cambio de hábitos e instrucción de higiene oral al padre y a la madre. Incluyó frecuencia y técnica de cepillado, tipo de cepillo (cabeza pequeña, cerdas rectas y blandas), crema dental (con flúor del tamaño de medio grano de arroz con cepillo seco) y uso de seda dental (por lo menos una vez al día). Se realizó el tratamiento odontológico en sillón en horas de la mañana durante varias citas. El comportamiento del niño fue levemente positivo durante las consultas. La atención se adaptó a las condiciones del niño (ayuda de los padres para posicionar al niño mientras se le realizaban los procedimientos debido a la talla). Se usó aislamiento relativo debido a la limitación de la apertura que no permitía la colocación de grapas. Se utilizaron medidas de seguridad para atender al niño (taco de mordida, algodones y gasas anudadas). Se utilizaron técnicas anestésicas infiltrativas y tronculares, cuyas dosis tuvieron en cuenta el peso. Se removieron las caries con cucharilla, se colocaron protector pulpar y restauraciones definitivas con resinas en molares (dientes 54, 64, 84 y 74 clasificación dental dígito dos) y formas plásticas en 51, 52 y 61, y se realizó pulido coronal. Se efectuaron controles a los 3 y 6 meses (figura 7). 
Fotos DURANTE Y AL FINAL DEL TRATAMIENTO. A Y B) ADAPTACIÓN. C) LIMITACiÓN DE LA APERTURA PARA

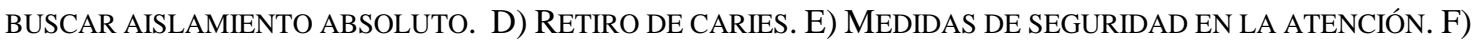

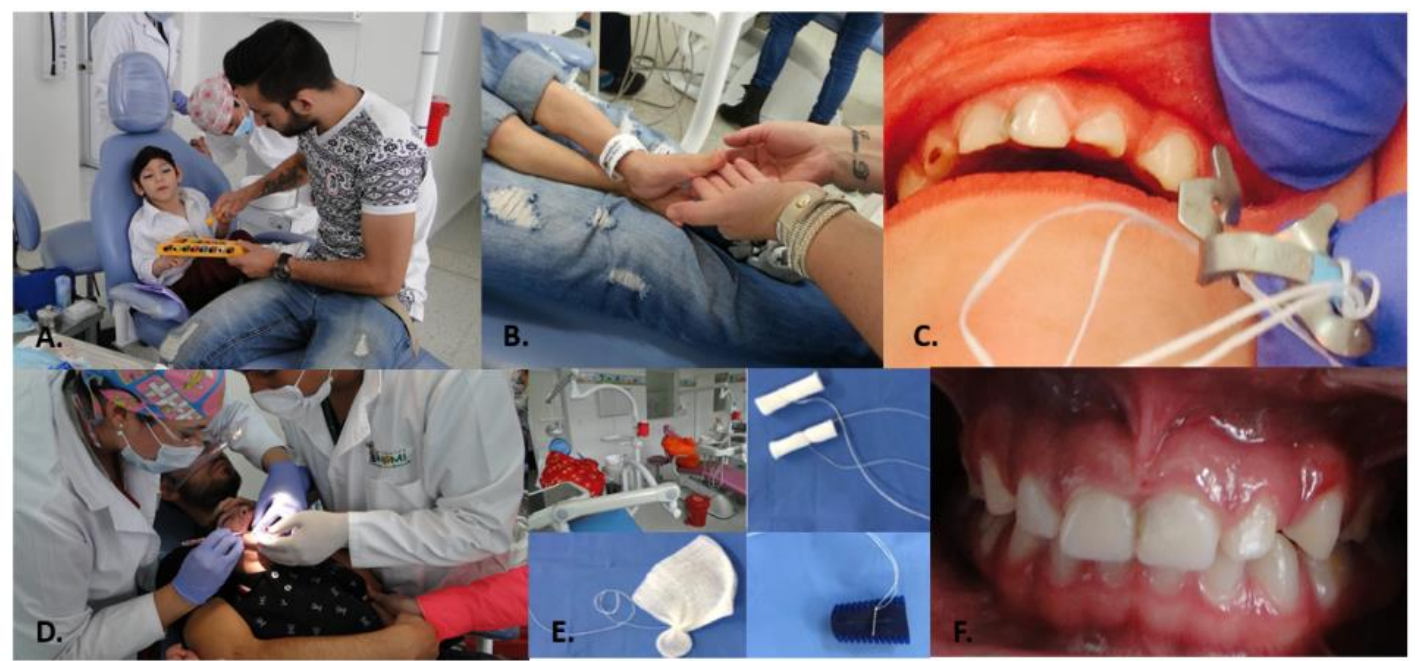

\section{DISCUSIÓN}

Los niños nacidos con microcefalia y bajo peso reciben atención por parte de un equipo multidisciplinario que incluye pediatría, enfermería, fisiatría, terapia ocupacional, terapia de lenguaje, nutrición, psicología, neurología, ortopedia, trabajo social, oftalmología, audiología y apoyo educacional para preescolar (11). El equipo analiza la importancia de la atención primaria en salud a este tipo de pacientes. Sin embargo, a menudo el(la) estomatólogo(a) u odontólogo(a) pediatra es invisible en el equipo multidisciplinario como se apreció en la guía de la National 
Guidance Alliance del Reino Unido y en el presente caso, pues el niño soló ingresó a consulta odontológica los 3 años y 7 meses para valoración.

Factores de riesgo prenatales como el nacimiento pretérmino y perinatales como el bajo peso al nacer se asocian a la IMOC. La IMOC en niños nacidos antes de 35 semanas se atribuye a hipoxia perinatal en $20 \%$ de los casos y no se atribuye a esta en el $12 \%$ (11). Como antecedentes de la madre en este caso se reportó en la historia clínica diabetes gestacional y preclamsia grave.

La microcefalia primaria está presente al nacimiento (12). La extensión del deterioro funcional a largo plazo se relaciona con la gravedad de la encefalopatía inicial. La mayor manifestación motora es del tipo discinético (movimientos involuntarios), mientras que el resto están relacionados con el tono, esto es, hipotonía (flacidez), espasticidad (rigidez) o distonía (tono fluctuante), así como control tardío del sostén cefálico, gateo y dificultades en la alimentación (11). En este caso se había asociaciones sistémicas como trastorno neurosensorial, alteraciones del tono muscular, defectos motores, discapacidad intelectual y convulsiones $(11,12)$. Además de la cuadriparesia espástica donde el niño tenía alteración del desarrollo motor con ausencia de sostén cefálico, sedestación (mantener la posición de sentado), bipedestación (mantenerse de pie) y marcha a los 3 años y 7 meses.

La asociación entre la microcefalia y el retardo en peso, talla y disminución del perímetro cefálico en 3 desviaciones estándar (12) se evidenció en este caso una talla de $82 \mathrm{~cm}$, un peso de $9,2 \mathrm{~kg} \mathrm{y}$ un perímetro cefálico de $34 \mathrm{~cm}$. Según los patrones de crecimiento de la OMS, para una edad de 3 años y 7 meses, el peso puntuación $Z$ en niños de 0 es $15,3 \mathrm{~kg}$, la talla puntuación $Z$ de 0 es de $101 \mathrm{~cm}$ y la puntuación $\mathrm{Z}$ de 0 en cuanto al perímetro cefálico es de $50 \mathrm{~cm}$ (13). Esto muestra 
claramente en este caso la asociación entre la microcefalia y patrones de crecimiento en niños con puntuación Z de -3 y por debajo de los valores reportados en Colombia para esta misma edad (14). El niño nació a las 28 semanas de gestación con un peso de 925 g, una talla de 34 cm y un perímetro cefálico de $25 \mathrm{~cm}$, lo que corrobora que uno de los diagnósticos para determinar microcefalia es 1 desviación estándar por debajo de 3 del promedio de normalidad (12) y de riesgo de IMOC por parto pretérmino antes de 32 semanas o bajo peso al nacer menor de $2500 \mathrm{~g} \mathrm{(10).}$

El bajo peso al nacer se ha relacionado con una baja densidad ósea, lo que incrementa el riesgo de fracturas $(11,15)$. Esto hace que, como consideración estomatológica (odontológica), se debe tener cuidado durante procedimientos como las extracciones dentales en la que se sostiene la mandíbula para evitar fracturas.

Otras asociaciones sistémicas de la IMOC se relacionan con el desarrollo del lenguaje, lo que genera dificultades en la comunicación, el lenguaje y el habla (11). La nula funcionalidad del habla fue evidente en este caso.

Rodrigues Santos et al. (16) en niños con IMOC identificaron una fuerte hipotonicidad de los músculos masticatorios, una disminución en la actividad muscular, presencia movimientos involuntarios y disminución en la actividad eléctrica que generaba alteraciones en la función masticatoria. De acuerdo con lo anterior, se requiere de un manejo postural al comer, así como de la adaptación de los alimentos (fluidos, texturas y sabores), con técnicas de alimentación en los que haya estimulación y modificación de la colocación de la cuchara (11). En concordancia con esto, se recomendó a los acudientes del niño una interconsulta con terapia miofuncional y del lenguaje. 
En el ámbito estomatológico se ha reportado en los niños con IMOC una asociación con desarmonía dentomaxilar, respiración oral, clase II esquelética, micrognatismo y retrognatismo mandibular, maloclusiones clase II, sobremordidas horizontal y vertical aumentadas, mordida abierta anterior con una prevalencia del $92 \%$, posicionamiento anterior y hacia abajo de la lengua y trauma dentoalveolar $(9,10)$. Otra manifestación es la sialorrea, lo cual indica la necesidad de evaluar otros factores como historial de medicación, reflujo y molestias dentales. Dependiendo del grado de sialorrea, el manejo será con toxina botulínica (11).

Un desgaste dental excesivo como consecuencia del bruxismo también se ha reportado en niños con IMOC. El tratamiento en estos casos es toxina botulínica en combinación con una placa neuromiorrelajante $(8,17)$.

Abanto et al. (8) explican que, a mayor grado de deterioro neurológico, mayor riesgo de enfermedades orales, mayor presencia de caries dental por consistencia en la dieta y dificultad del cuidador para realizar la higiene oral al menor (10). Gutiérrez et al. (18) describieron un aumento en las lesiones por caries en estadios iniciales, ICDAS 2 y 3, en niños que habían recibido tratamiento para la xialorrea por bajo flujo salival y mayor osmolalidad salival. Bolaños-Sánchez (19) señala la importancia de usar criterios de evaluación adecuados, como el ICDAS, que ayuda a comprender mejor la situación de caries en esta población y a desarrollar programas preventivos para personas de riesgo alto. En el caso aquí descrito el uso de ICDAS permitió detectar lesiones de caries en estadios iniciales lo que permitió llevar a cabo un programa preventivo individualizado. La prevención de la caries dental a edades tempranas es importante para evitar sintomatología en la niñez y la adolescencia como la referida por 
Akhter et al. (20) que consistía en dolor dental y dificultad en la alimentación, lo que afecta la calidad de vida de los niños con IMOC (20).

En este caso se identificó falta de consejería dietaria dado que el niño tenía habito de biberón de día y de noche, a raíz del cual se desarrolló caries de la infancia temprana. Este tipo de caries se asocia a la dieta y la falta de función oromotora que aumentan el riesgo de caries dental en niños con IMOC (21).

La atención estomatológica de niños con microcefalia, bajo peso al nacer y cuadriparesia espástica se debe enfocar a programas de adaptación a la cita odontológica, valoración de riesgo de caries, consejería dietaria, promoción y prevención de la caries dental y otras patologías, previa realización de una exhaustiva historia clínica, en la que se consignen todos los antecedentes médicos y medicamentos suministrados al paciente. Asimismo, se debe realizar un consentimiento informado y un adecuado manejo de comportamiento para disminuir la ansiedad durante la consulta y el tratamiento (10,22-24). Tampoco se debe olvidar el manejo de medidas de seguridad en la atención de los niños con IMOC, como colocar cojines debajo de las extremidades, anudar con seda dental, colocar grapas, algodones y bloques de mordida, y evitar que muerdan la boquilla plástica del eyector, pues la pueden broncoaspirar.

La higiene oral en niños con IMOC debe individualizarse mediante instrucciones a los padres o cuidadores. Se les debe dar a conocer la existencia de diferentes aditamentos como abrebocas, bloques de mordida, cepillos eléctricos y portasedas que facilitan una adecuada remoción de placa bacteriana (22-24). 
El(la) estomatólogo(a) u odontólogo(a) pediatra es esencial en la atención de los niños con IMOC para prevenir ciertas condiciones orales o reducir complicaciones orales. Por eso debe ser parte del equipo multidisciplinario y contribuir con el bienestar de estos niños, tal y como lo reportan Santacruz et al. (25) y Parakh et al. (26).

\section{CONCLUSIONES}

En este reporte de caso se cumplió con todos los objetivos planteados para la atención integral. Se realizaron los respectivos controles que mostraron compromiso por parte de los padres para cambiar los hábitos de riesgo de caries dental y adherirse a las técnicas e instrucciones de higiene oral para mantener una óptima salud bucal y mejorar la calidad de vida del niño. La atención estomatológica de niños con microcefalia, bajo peso al nacer y cuadriparesia espástica se debe enfocar según las condiciones y necesidades específicas de cada niño. Se debe enfatizar el mantenimiento de la salud bucal después de cualquier tratamiento odontológico, de allí la importancia de realizar una buena higiene oral al niño por parte del cuidador.

\section{RECOMENDACIONES}

Debe promoverse la participación del(la) estomatólogo(a) u odontólogo(a) pediatra en el equipo multidisciplinario para la atención estomatológica de niños con IMOC. Para ello se debe replantear cómo se conforman dichos grupos así como el contenido de las guías de salud para incluir la salud bucal en este tipo de pacientes. 


\section{REFERENCIAS}

1. World Health Organization (WHO). Born too soon the global action report on preterm birth. Geneva, Switzerland: WHO; 2012.

2. Robaina Castellanos G, Ruiz Tellachea Y, Domínguez Dieppa F, Roca Molina MC, Riesgo Rodríguez S, Berdayes Millián JD. Neurodesarrollo en recién nacidos ventilados con menos de 1500 gramos. Rev Cub Pediatr. 2015; 72(4): 267-74.

3. Agency for Clinical Innovation. Management of cerebral palsy in children: a guide for allied health professionals. (GL2018_006). North Sydney, Australia; New South Wales Ministry of Health; 2018. Available at: https://www1.health.nsw.gov.au/pds/ActivePDSDocuments/GL2018_006.pdf

4. Malagón Valdez J. Parálisis cerebral. Medicina (Buenos Aires). 2015; 67(6): 586-92.

5. Ardila S, Espinosa E. Caracterización clínica y radiológica de pacientes pediátricos con malformaciones de corteza cerebral en dos hospitales de Bogotá periodo 2007-2010. Bogotá, Colombia: Universidad Militar Nueva Granada; 2010.

6. Nazer H J, Cifuentes O L. Malformaciones congénitas en Chile y Latino América: Una visión epidemiológica del ECLAMC del período 1995-2008. Rev Med Chile. 2011; 139(1): 72-8.

7. Pachajoa H, Ariza Y, Isaza C, Méndez F. Defectos congénitos mayores en un hospital de tercer nivel en Cali, Colombia 2004-2008. Rev Salud Publica (Bogotá). 2011; 13(1): 152-62.

8. Abanto J, Ortega AO, Raggio DP, Bönecker M, Mendes FM, Ciamponi AL. Impact of oral diseases and disorders on oral-health-related quality of life of children with cerebral palsy. Spec Care Dentist. 2014 Mar-Apr; 34(2): 56-63.

9. Barrionuevo NL, Solís FF. Anomalías dentó maxilares y factores asociados en niños con parálisis cerebral. Rev Chil Pediatr. 2008; 79(3): 272-80. 
10. Sehrawat N, Marwaha M, Bansal K, Chopra R. Cerebral palsy: a dental update. Int J Clin Pediatr Dent. 2014; 7(2): 109-18.

11. National Guidance Alliance (UK). Cerebral palsy in the under-25s: assessment and management. London, UK: National Institute for Health and Care Excellence. 2017 Jan. Available at: https://www.ncbi.nlm.nih.gov/books/NBK419326/

12. Martí Herrero M, Cabrera López JC. Macro- y microcefalia. Trastornos del crecimiento craneal. (Protocolos diagnósticos y terapéuticos en Pediatría). Madrid, España: Asociación Española de Pediatría; 2008. Disponible en: https://www.aeped.es/sites/default/files/documentos/25$\underline{\text { macromicrocefalia.pdf }}$

13. Organización Mundial de la Salud (OMS). Patrones de crecimiento infantil. Puntuación Z niños OMS. Ginebra, Suiza: OMS; 2018.

14. López Rodríguez YN. Antropometría craneofacial en niños de 0 a 4 años. Una perspectiva Bayesiana. Bogotá, Colombia: Universidad Nacional de Colombia, Repositorio Institucional; 2015. Disponible en: https://repositorio.unal.edu.co/handle/unal/54052

15. Díaz AT. Estudio de la densidad ósea en niños y adolescentes con afectación moderada y severa por parálisis cerebral. (Tesis doctoral). Sevilla, España: Universidad de Sevilla; 2016. Disponible en: https://idus.us.es/xmlui/handle/11441/39821

16. Rodrigues Santos MTB, Guare RO, Celiberti P, Siqueira WL. Caries experience in individuals with cerebral palsy in relation to oromotor dysfunction and dietary consistency. Special Care Dent. 2009; 29(5): 198.

17. Santos MTBR, Manzano FS, Genovese WJ. Different approaches to dental management of self-inflicted oral trauma: oral shield, botulinum toxin type A neuromuscular block, and oral surgery. Quintessence Int. 2008 Feb; 39(2): e63-69. 
18. Gutierrez GM, Siqueira VL, Loyola-Rodriguez JP, Diniz MB, Guaré RO, Ferreira AC, Santos MT. Effects of treatments for drooling on caries risk in children and adolescents with cerebral palsy. Med Oral Patol Oral Cir Bucal. 2019 Mar 1; 24(2): 204-10.

19. Bolaños-Sánchez J. Caries de aparición temprana en infantes con parálisis cerebral espástica: ventajas de usar el sistema internacional de detección y evaluación de caries ICDAS. KIRU. 2015 ene-jun; 12(1): 74-8.

20. Akhter R, Hassan NMM, Martin EF, Muhit M, Smithers-Sheedy H, Badawi N, Khandaker G. Caries experience and oral health-related quality of life (OHRQoL) of children and adolescents with cerebral palsy in a low-resource setting. BMC Oral Health. 2019; 19(1): 15.

21. Santos MT, Guare RO, Celiberti P, Siqueira WL. Caries experience in individuals with cerebral palsy in relation to oromotor dysfunction and dietary consistency. Spec Care Dentist. 2009 Oct; 29(5): 198-203.

22. Guideline on management of dental patients with special health care needs. Pediatr Dent. 2016 Oct 15; 38(5): 67-72.

23. Ministerio de Salud. Guía clínica. Salud oral integral para menores de 20 años en situación de discapacidad que requieren cuidados especiales en odontología. Santiago, Chile: Minsal; 2012.

24. Martínez H, Treviño M, Rivera G. Guía para el cuidado de la salud oral en pacientes con necesidad de cuidados especiales de salud en México. Revista ADM. 2011; LXVIII(5): 2228.

25. Santacruz HDL, Molinar YH, Sandoval BEM, Berber MÁR, Delgadillo GT. Estrategias terapéuticas de calidad en odontopediatría: parálisis cerebral. Acta Pediatr Méx. 2019 ene 28; 40(1): 32-43. 
26. Parakh A, Singh R, Bhat D, Kulkarni N, Fernandes G. A Mini review on cerebral palsy and its implications in dentistry. OBDS. 2018 dic 10; 01-7. 